\title{
Practical identifiability of HIV dynamics models
}

\author{
J. Guedj, ${ }^{1,2, *}$ R. Thiébaut ${ }^{1,2}$ and D. Commenges ${ }^{1,2}$ \\ ${ }^{1}$ INSERM, U875 (Biostatistique), Bordeaux, F-33076, FRANCE \\ ${ }^{2}$ Université Victor Segalen Bordeaux 2, Bordeaux, F-33076, FRANCE
}

\section{Abstract}

We study the practical identifiability of parameters, i.e the accuracy of the estimation that can be hoped, in a model of HIV dynamics based on a system of non-linear Ordinary Differential Equations (ODE). This depends on the available information such as the schedule of the measurements, the observed components, and the measurement precision. The number of patients is another way to increase it by introducing an appropriate statistical "population" framework. The impact of each improvement of the experimental condition is not known in advance but it can be evaluated via the Fisher Information Matrix (FIM). If the non-linearity of the biological model, as well as the complex statistical framework makes computation of the FIM challenging, we show that the particular structure of these models enables to compute it as precisely as wanted. In the HIV model, measuring HIV viral load and total CD4+ count were not enough to achieve identifiability of all the parameters involved. However, we show that an appropriate statistical approach together with the availability of additional markers such as

*email: jg1@isped.u-bordeaux2.fr 
infected cells or activated cells should considerably improve the identifiability and thus the usefulness of dynamical models of HIV.

Key Words: HIV dynamics, Non-linear differential equations, Parameter estimation, Practical identifiability, Fisher information matrix, Design. 


\section{Introduction: general}

Since the early 1990s, the introduction of non-linear systems of Ordinary Differential Equations (ODE) to model the interaction between the Human Immunodeficiency Virus (HIV) and the immune system have brought critical insights on the disease (Ho et al., 1995; Perelson et al., 1996; Perelson, 2002; $\mathrm{Wu}, 2005)$. Nevertheless, these works have been often limited by the difficulty to handle models defined by non-linear ODE in which the identifiability of the involved parameters is difficult to assess.

The concept of identifiability encompasses two notions: the theoretical identifiability and the practical identifiability. The theoretical identifiability is a precisely defined concept of binary concept (see section 2.4); the practical identifiability is less clearcut and may be defined as the ability to estimate a given set of parameters with an accuracy considered as satisfactory according to the context of the study. A very clear explanation of the difference between these concepts is given in Petersen et al. (2001): "the theoretical identifiability is based on the model structure and the available measured outputs, and gives an indication of the maximum amount of information that can be obtained from a theoretical experiment. The practical identifiability on the contrary not only depends on the model structure, but is also related to the experimental conditions". Consequently even if theoretical identifiability is assessed, the practical identifiability is not granted. It can be noted that the theoretical identifiability in non-linear systems of ODE has been deeply studied (Pohjanpalo, 1978; Holmberg, 1982; Julien et al., 1997; Audoly et al., 2001; Xia and Moog, 2003) and this issue is out of the scope of this paper.

To improve the practical identifiability, one can increase the available 
information via the number of measurements, the number of observed components or the precision of the measurements instruments. One of the main interest is to quantify the supply of each type of information in the improvement of the accuracy of the parameter estimation.

Classically, once data are collected, they are analyzed using simple statistical methods, such as non-linear regression made separately patient-bypatient (Perelson et al., 1996; Stafford et al., 2000; Ribeiro et al., 2002). As noted in $\mathrm{Wu}(2005)$ in the framework of the HIV dynamics models and more generally in Davidian and Giltinian (1995) and Pinheiro and Bates (2000), a major improvement for the practical identifiability is to take advantage of the whole available information, in particular the between-subject variation: the parameters may vary from one subject to another while being considered as realisations from the same distribution (Putter et al., 2002, Banks et al., 2005; Huang and Wu, 2006; Huang et al., 2006; Bortz and Nelson, 2006; Guedj et al., 2007). Such models are in the framework of Non-Linear Mixed Effect models (NLME). The number of patients included in the statistical analysis is consequently another potential way to increase information.

Whatever the approach (patient-by-patient or population), the use of the Fisher Information Matrix (FIM) to check and to measure the practical identifiability of parameters estimated by Maximum Likelihood Estimation (MLE) is natural, since the MLE are asymptotically efficient with an asymptotic variance given by the inverse of the FIM. In a population and non-linear framework, the FIM has no closed-form and its computation involves multidimensional integrals, becoming a challenging issue. That is why some authors have suggested in other contexts to make simplifications of it but this can 
lead to a lack of precision (Retout et al., 2001), especially in the particularly complex context of ODE models. Thus, we propose a way to compute the FIM as precisely as wanted through the "exact" computation of the scores, thanks to the use of the Louis' formula as well as to the introduction of the sensitivity equations. The methods is then applied to check the practical identifiability of parameters of an ODE system of HIV infection according to different observational designs.

The paper is organized as follows: in section 2, we develop the rationale of using the information matrix for studying practical identifiability. We show how it can be computed with good accuracy. In section 3, we describe an HIV dynamics model based on five equations. In section 4, we investigate the identifiability of the system for different existing observational designs varying according to the schedules of measurements, the components that can be measured and the population or the patient-by-patient approach.

\section{Statistical matrix and Fisher Information Matrix}

\subsection{General model for the system}

Let us consider a model of ODE for a population of $n$ subjects. For subject $i$ with $i=1, \ldots n$, this can be written:

$$
\left\{\begin{array}{l}
\frac{d \boldsymbol{X}^{(i)}(t)}{d t}=f\left(\boldsymbol{X}^{(i)}(t), \boldsymbol{\xi}^{(i)}\right) \\
\boldsymbol{X}^{(i)}(0)=h\left(\boldsymbol{\xi}^{(i)}\right)
\end{array}\right.
$$

where $\boldsymbol{X}^{(i)}(t)=\left(X_{1}^{(i)}(t), \ldots, X_{K}^{(i)}(t)\right)^{\prime}$ is the vector of the $K$ state variables. We write $\boldsymbol{X}\left(t, \boldsymbol{\xi}^{(i)}\right)=\boldsymbol{X}^{(i)}(t)$ to underline that $\boldsymbol{\xi}^{(i)}$ completely determines the trajectories $\boldsymbol{X}^{(i)}(t)$ where $\boldsymbol{\xi}^{(i)}=\left(\xi_{1}^{(i)}, \ldots, \xi_{p}^{(i)}\right)^{\prime}$ (' for transpose) is a vector of $p$ individual biological parameters which appear in the ODE system.

It is current to reparametrize the original system by a set of one-to-one 
transformations $\Psi_{l}, l=1 \ldots p$, to take into account the constraints that may exist for the parameters; namely the positivity of the biological parameters and that of an in vivo treatment effect comprised between 0 (total inefficacy) and 1 (perfect efficacy). Moreover we take into account the between-subject variation and thus we consider the $\boldsymbol{\xi}^{(i)}$ 's as the realization of random effects. In this approach, called in the following the "population approach", each patient has a different value for the biological parameters, but the variation from one subject to one other belongs to a general statistical framework:

$$
\left\{\begin{array}{l}
\tilde{\xi}_{l}^{(i)}=\Psi_{l}\left(\xi_{l}^{(i)}\right), \\
\tilde{\xi}_{l}^{(i)}=\phi_{l}+a_{l l} u_{l}^{(i)}, \quad l \leq p
\end{array}\right.
$$

We assume that only a subset of $q$ (with $q \leq p$ ) parameters will have a non-null variance and thus we may restrict to consider an independent qdimensional vector $\boldsymbol{u}^{(i)} \sim \mathcal{N}\left(0, I_{q}\right)$. This assumption could be relaxed without difficulty for introducing correlations between the random effects.

\subsection{Model for the observations}

The $M$ observed HIV markers are not directly the components of the biological system (1), but rather observation of combinations of the components of the original system. We ensure a Gaussian framework and homoskedasticity of the error measurements by working on transformed observations. In summary, let $Y_{i j m}$ denote the $j$ th measurements of the $m$ th observable component in subject $i$ at time $t_{j m}$ :

$$
Y_{i j m}=g_{m}\left(X\left(t_{j m}, \tilde{\boldsymbol{\xi}}^{(i)}\right)\right)+\epsilon_{i j m} \quad j=1, \ldots n_{m}, \quad m=1, \ldots, M
$$

where the $\epsilon_{i j m}$ are independent Gaussian measurement errors with zero mean and variances $\sigma_{m}^{2}$ and functions $g_{m}(\cdot), m=1, \ldots, M$ of $\mathbb{R}^{K}$ to $\mathbb{R}$ are assumed 
to be twice differentiable: the $g_{m}(\cdot)$ will be called the observable components. Notice that the sets of the measurement times (the schedule) $S=\left\{t_{j m}\right\}$ and of the observable components $G=\left\{g_{m}(\cdot)\right\}$ constitutes the design $\mathcal{D}_{S, G}$.

Moreover, the possibility of censored measurements may be easily taken into account; this is particularly relevant for HIV-RNA whose assays used to quantify it are limited by a detection threshold that may lead to undetectable values when the true viral load is below this threshold.

It can be noted that the above population model defines a framework where the patients are independent and identically distributed (i.i.d). This assumption could be relaxed when introducing explanatory variables in the regression model (2) or considering variable design between patients in the model for the observations (3).

\subsection{Log-Likelihood and introduction of the FIM}

The set of parameters of interest is $\boldsymbol{\theta}=\left(\left(\phi_{l}\right)_{l \leq p}, \boldsymbol{A}=\left(a_{l l}\right)_{l \leq q}, \boldsymbol{\sigma}=\left(\sigma_{l}\right)_{l \leq M}\right)$. Let $\mathcal{F}_{i}$ denote the full information given by both the possibly censored observation of $\boldsymbol{Y}_{i}$ and $\boldsymbol{u}^{(i)}$ (in probabilist terms, the sigma-field generated by the possibly censored observation of $\boldsymbol{Y}_{i}$ and $\left.\boldsymbol{u}^{(i)}\right)$. We denote by $\mathcal{L}_{\mathcal{F}_{i} \mid \boldsymbol{u}^{(i)}}(\theta)$ (noted only $\mathcal{L}_{\mathcal{F}_{i} \mid \boldsymbol{u}^{(i)}}$ in the following) the full individual likelihood given the random effects in a population model (Commenges et al., 2006). In the case where there are no left-censored data, the i.i.d Gaussian terms for the error measurements lead to a straightforward expression for $\mathcal{L}_{\mathcal{F}_{i} \mid \boldsymbol{u}^{(i)}}$ :

$$
\mathcal{L}_{\mathcal{F}_{i} \mid \boldsymbol{u}^{(i)}}=\prod_{j, m} \frac{1}{\sigma_{m} \sqrt{2 \pi}} \exp \left[-\frac{1}{2}\left(\frac{Y_{i j m}-g_{m}\left(X\left(t_{j m}, \tilde{\boldsymbol{\xi}}^{(i)}\right)\right)}{\sigma_{m}}\right)^{2}\right]
$$

We use a solver for stiff systems of ODE developed in Radhakrishnan and Hindmarsh (1993) for computing the $\boldsymbol{X}\left(t_{j m}\right)$ 's; the required relative precision 
is equal to $10^{-11}$. The code is written in Fortran. The likelihood for the case with left-censored observations due to detection limits is given explicitly in Thiébaut et al. (2006). Let us denote by $\mathcal{O}_{i}$ the information brought by the observation of $Y_{i}$ (possibly censored). We denote by $\mathcal{L}_{\mathcal{O}_{i}}(\theta)$ (noted only $\mathcal{L}_{\mathcal{O}_{i}}$ ) the observed individual likelihood. It is obtained from $\mathcal{L}_{\mathcal{F}_{i} \mid \boldsymbol{u}^{(i)}}$ as:

$$
\mathcal{L}_{\mathcal{O}_{i}}=\int_{\mathbb{R}^{q}} \mathcal{L}_{\mathcal{F}_{i} \mid \boldsymbol{u}^{(i)}} \varphi(\mathbf{u}) d \mathbf{u}
$$

where $\varphi$ is the multivariate normal density of $N\left(0, I_{q}\right)$. We will denote by $L_{\mathcal{F}_{i} \mid \boldsymbol{u}^{(i)}}=\log \mathcal{L}_{\mathcal{F}_{i} \mid \boldsymbol{u}^{(i)}}$ and $L_{\mathcal{O}_{i}}=\log \mathcal{L}_{\mathcal{O}_{i}}$ the full (given random effects) and observed individual log-likelihoods, respectively. The global observed log-likelihood is $L_{\overline{\mathcal{O}}_{n}}(\theta)=\sum_{i=1, \ldots, n} L_{\mathcal{O}_{i}}$ by independence between patients.

The elementary FIM for the patient $i$ is given as

$$
\mathcal{I}_{\mathcal{D}, i}(\boldsymbol{\theta})=\mathbb{E}_{\boldsymbol{\theta}}\left(-\frac{\partial^{2} L_{\mathcal{O}_{i}}(\boldsymbol{\theta})}{\partial \boldsymbol{\theta} \partial \boldsymbol{\theta}^{T}}\right)
$$

and we can define the FIM for the whole sample of $n$ patients:

$$
\mathcal{I}_{\mathcal{D}, n}(\boldsymbol{\theta})=\mathbb{E}_{\boldsymbol{\theta}}\left(-\frac{\partial^{2} L_{\overline{\mathcal{O}}_{n}}(\boldsymbol{\theta})}{\partial \boldsymbol{\theta} \partial \boldsymbol{\theta}^{T}}\right)
$$

\subsection{Fisher Information Matrix and theoretical local identifiability}

Let us consider the problem of (local) identifiability for the parameters as defined notably in Rothenberg (1971). Let $\mathbf{Y}$ be a random vector with a probability density function (p.d.f.) $\varphi\left(y, \boldsymbol{\theta}_{0}\right)$, and let $A=V\left(\boldsymbol{\theta}_{0}\right)$ be an open neighborhood of $\boldsymbol{\theta}_{0}$. A parameter point $\boldsymbol{\theta}_{0}$ is said to be locally identifiable if there is no other point $\boldsymbol{\theta} \in A$ which is observationally equivalent, that is for which $\psi(\boldsymbol{y}, \boldsymbol{\theta})=\psi\left(\boldsymbol{y}, \boldsymbol{\theta}_{0}\right)$ for all $\boldsymbol{y}$. Under weak assumptions, Rothenberg (1971) shown that a necessary and sufficient condition for local 
identifiability in $\boldsymbol{\theta}_{0}$ is that $\mathcal{I}_{\mathcal{D}, n}(\boldsymbol{\theta})$ is invertible for all $\boldsymbol{\theta} \in A$. This property is not straightforward to assess in non-linear systems. It can be noted however that $\mathcal{I}_{\mathcal{D}, n}\left(\boldsymbol{\theta}_{0}\right)$ invertible is a necessary but not a sufficient condition for local identifiability.

If $\mathcal{I}_{\mathcal{D}, n}\left(\boldsymbol{\theta}_{0}\right)$ is not invertible, one may analyze the lowest eigenvalues of $\mathcal{I}_{\mathcal{D}, n}\left(\boldsymbol{\theta}_{0}\right)$. The elements of the associated eigenvectors may suggest simplifications or reparametrizations of the initial statistical model as explained in details in Vajda et al. (1989); the other possibility, that we apply in the following, is to fix one of the concerned parameters according to values found in the literature.

\subsection{Information matrix and practical identifiability}

Theoretical identifiability is not sufficient for the practical use of these models. We wish to know whether we can estimate the set $\boldsymbol{\theta}$ of parameters of interest of the model with sufficient accuracy with a given design at a point value $\boldsymbol{\theta}=\boldsymbol{\theta}_{0}$. For this aim, the information matrix is of crucial importance. From the Cramer-Rao bound (see for instance Knight, 2001) we know that the inverse of the FIM is the lower bound of the variance-covariance matrix of any unbiased estimator. Moreover, the study of the asymptotic distribution of the Maximum Likelihood Estimator (MLE) $\hat{\boldsymbol{\theta}}$ allows considering that, if there is sufficient information ( $n$ large enough in the population approach or $n_{i m}$ in the patient-by-patient approach, see below), the variance of $\hat{\boldsymbol{\theta}}$ is approximately $\mathcal{I}_{\mathcal{D}, n}^{-1}(\boldsymbol{\theta})$. So the accuracy attainable with design $\mathcal{D}$ can be measured by $\mathcal{I}_{\mathcal{D}, n}^{-1}(\boldsymbol{\theta})$ and for a given parameter $\theta_{j}$, we can contemplate the

standard deviation of its estimator $s e_{\mathcal{D}, n, j}(\hat{\boldsymbol{\theta}})=\sqrt{\mathcal{I}_{\mathcal{D}, n, j j}^{-1}(\boldsymbol{\theta})}$ (where "se" stands for standard error) or, for strictly positive parameters, the relative 
error $r e_{\mathcal{D}, j}(\hat{\boldsymbol{\theta}})=\sqrt{\mathcal{I}_{\mathcal{D}, n, j j}^{-1}(\boldsymbol{\theta})} / \theta_{j}$. This relative error is sometimes called the coefficient of variation.

As discussed in section 2.1., it is advantageous to reparametrize the biological parameters, often in term of $\tilde{\boldsymbol{\xi}}^{(i)}=\log \left(\boldsymbol{\xi}^{(i)}\right)$. First it enables to take the constraint of positivity into account; moreover the log-transform allows to obtain a model which has an intrinsic information matrix, in the sense that it does not depend on the measurements units. Moreover, by use of the $\delta$-method, the standard error of the estimator of the log-transformed parameter $\hat{\phi}_{l}$ is asymptotically equal to the relative error of the parameter in its natural scale.

Thus, when computing $\mathcal{I}_{\mathcal{D}, n}(\boldsymbol{\theta})$ at a point value $\theta=\theta_{0}$, one can directly interpret the standard errors of the estimators of the log-transformed biological parameters: for instance a standard error of 0.1 means that we know only one significant number and the parameter is poorly identifiable; a standard error larger than 1 for one biological parameter tells us that we only know the order of magnitude of the original parameter and we consider that $\boldsymbol{\theta}$ is not practically identifiable for the value $\boldsymbol{\theta}=\boldsymbol{\theta}_{0}$. In this case, one can still study the eigenvalues and the eigenvectors of the FIM as discussed above to determine the origin of the practical non-identifiability. Let us note that the log-transformation is particularly adapted in these models where difficulties often come from combinations such as $\frac{\xi_{i}}{\xi_{j}}$ and $\xi_{i} \xi_{j}$.

\subsection{Criteria for comparing different designs}

Several real-valued criteria have been introduced to facilitate comparison of designs. A general class of criteria is defined in Kiefer (1974). One of the most widely used is the $D$-criteria defined as $\operatorname{det}\left(\mathcal{I}_{\mathcal{D}, n}(\boldsymbol{\theta})\right)$ or as its loga- 
rithm $\phi_{n}(\mathcal{D})=\ln \left(\operatorname{det}\left(\mathcal{I}_{\mathcal{D}, n}(\boldsymbol{\theta})\right)\right)$. The bigger $\phi_{n}(\mathcal{D})$ (noted only $\phi(\mathcal{D})$ in the following) is, the better it is since it means that we reduce the volume of the confidence ellipsoid to obtain more global accurate coefficients (Box and Draper, 1959). This criterion has many advantages like its simplicity and its invariance by non degenerated transformations on the parameters (Fedorov, 1972).

A traditional measure for comparing the D-efficiency of two designs $\mathcal{D}$ and $\mathcal{D}^{\prime}$ with $\phi(\mathcal{D}) \leq \phi\left(\mathcal{D}^{\prime}\right)$ is the standardized ratio:

$$
\mathcal{E}_{\mathcal{D}, \mathcal{D}^{\prime}, n}(\boldsymbol{\theta})=\exp \left\{\frac{1}{2 * \operatorname{dim}(\boldsymbol{\theta})}\left[\phi\left(\mathcal{D}^{\prime}\right)-\phi(\mathcal{D})\right]\right\}
$$

$\mathcal{E}_{\mathcal{D}, \mathcal{D}^{\prime}, n}(\boldsymbol{\theta})$ (noted only $\mathcal{E}_{\mathcal{D}, \mathcal{D}^{\prime}}$ in the following) measures the expected (geometric) mean factor diminution of the standard deviation when using $\mathcal{D}^{\prime}$ compared to $\mathcal{D}$. Then $100 *\left(1-\mathcal{E}_{\mathcal{D}, \mathcal{D}^{\prime}}\right)$ expresses the variation as a percentage. In the same idea, one can introduce $\mathcal{E}_{\mathcal{D}}=\exp \left\{\frac{1}{2 * \operatorname{dim}(\boldsymbol{\theta})}[-\phi(\mathcal{D})]\right\}: \mathcal{E}_{\mathcal{D}}$ would be equal to the geometric mean value of the expected standard deviations of the estimators of $\boldsymbol{\theta}$ according to a design $\mathcal{D}$ if the estimator were independent.

To compare two nested structures or to focus on a particular subset of parameters, one can use the $D_{s}$-efficiency. Let us partition $\boldsymbol{\theta}$ into $\left(\boldsymbol{\theta}_{1}, \boldsymbol{\theta}_{2}\right)$ with $\operatorname{dim}\left(\boldsymbol{\theta}_{1}\right)=s$ and the FIM into

$$
\mathcal{I}(\boldsymbol{\theta})=\left(\begin{array}{ll}
\mathcal{I}_{11} & \mathcal{I}_{12} \\
\mathcal{I}_{12} & \mathcal{I}_{22}
\end{array}\right) \text { with } \mathcal{I}_{11} \text { a matrix of dimension }(s, s)
$$

One can study as upper $\phi_{s}(\mathcal{D})=\ln \left(\operatorname{det}\left(\mathcal{I}_{11}^{(2)}\right)\right)$ with $\mathcal{I}_{11}^{(2)}=\left(\mathcal{I}_{11}-\mathcal{I}_{12} \mathcal{I}_{22}^{-1} \mathcal{I}_{12}^{T}\right)$. Note that it is necessary that $\mathcal{I}_{22}$ is invertible. 
2.7 Computation of the information matrix in population models defined by a system of $O D E$

Let us define the individual scores

$$
\boldsymbol{U}_{\mathcal{F}_{i} \mid \boldsymbol{u}^{(i)}}(\boldsymbol{\theta})=\frac{\partial L_{\mathcal{F}_{i} \mid \boldsymbol{u}^{(i)}}(\boldsymbol{\theta})}{\partial \boldsymbol{\theta}} \text { and } \boldsymbol{U}_{\mathcal{O}_{i}}(\boldsymbol{\theta})=\frac{\partial L_{\mathcal{O}_{i}}(\boldsymbol{\theta})}{\partial \boldsymbol{\theta}}
$$

respectively the score given the random effects and the observed score. We have shown in Guedj et al. (2007) that a semi-analytic expression for $U_{\mathcal{F}_{i} \mid \boldsymbol{u}^{(i)}}$ could be obtained when using the particular structure of these models. The reader can refer to the Appendix to obtain more details about the methodology. Using the Louis' formula (Louis, 1982), one can link the score given the random effects and the observed score :

$$
\boldsymbol{U}_{\mathcal{O}_{i}}=\left(\mathcal{L}_{\mathcal{O}_{i}}\right)^{-1} \int_{\mathbb{R}^{q}} \mathcal{L}_{\mathcal{F}_{i} \mid \boldsymbol{u}^{(i)}} \boldsymbol{U}_{\mathcal{F}_{i} \mid \boldsymbol{u}^{(i)}}(\mathbf{u}) \varphi(\mathbf{u}) d \mathbf{u}
$$

The integrals can be computed by adaptive Gaussian quadrature using a routine developed in Genz and Keister (1996) and $\boldsymbol{U}_{\mathcal{O}_{i}}$ can be computed as precise as wanted (for $q=3$ we found that 100 nodes was sufficient to achieve an absolute precision of $10^{-3}$ ). The elementary FIM for the patient $i$ can be then deduced from its individual score:

$$
\mathcal{I}_{\mathcal{D}, i}(\boldsymbol{\theta})=\mathbb{E}_{\boldsymbol{\theta}}\left[\boldsymbol{U}_{\mathcal{O}_{i}}(\boldsymbol{\theta}) \boldsymbol{U}_{\mathcal{O}_{i}}(\boldsymbol{\theta})^{T}\right]
$$

Here, we have considered a model in which the $n$ patients are independent and identically distributed (see section 2.2.); the elementary FIM is the same for all the patients and we can define the generic elementary FIM:

$$
\mathcal{I}_{\mathcal{D}}(\boldsymbol{\theta})=\mathbb{E}_{\boldsymbol{\theta}}\left[\boldsymbol{U}_{\mathcal{O}_{i}}(\boldsymbol{\theta}) \boldsymbol{U}_{\mathcal{O}_{i}}(\boldsymbol{\theta})^{T}\right]
$$

The FIM for the whole sample is deduced from the generic individual FIM and $\mathcal{I}_{\mathcal{D}, n}(\boldsymbol{\theta})=n \mathcal{I}_{\mathcal{D}}(\boldsymbol{\theta})$. However, $\mathcal{I}_{\mathcal{D}}(\boldsymbol{\theta})$ defined by (6) has no analytical 
expression. Nevertheless it can be evaluated by simulation. First it requires to generate data under the probability $P_{\theta}$, i.e. to replicate $N$ patients (with $N$ large) having the same distribution law than that for the elementary patient. One can thus get an approximation of expression (6) by:

$$
\mathcal{I}_{\mathcal{D}}^{N}(\boldsymbol{\theta})=\frac{1}{N} \sum_{j=1, \ldots, N} \boldsymbol{U}_{\mathcal{O}_{j}}(\boldsymbol{\theta}) \boldsymbol{U}_{\mathcal{O}_{j}}^{T}(\boldsymbol{\theta})
$$

By of the law of large numbers we have that $\mathcal{I}_{\mathcal{D}}^{N}(\boldsymbol{\theta})$ tends toward $\mathcal{I}_{\mathcal{D}}(\boldsymbol{\theta})$ as $N$ tends toward infinity, so that the approximation error can be made arbitrarily small (The program is available from the corresponding author).

One can check that $N$ is large enough by computing $C=N^{-1} U_{\overline{\mathcal{O}}_{N}}^{T}(\theta)\left[\mathcal{I}_{\mathcal{D}}^{N}(\boldsymbol{\theta})\right]^{-1}(\boldsymbol{\theta}) \boldsymbol{U}_{\overline{\mathcal{O}}_{N}}(\boldsymbol{\theta})$ where $\boldsymbol{U}_{\overline{\mathcal{O}}_{N}}=\sum_{j=1, \ldots, N} \boldsymbol{U}_{\mathcal{O}_{j}}(\boldsymbol{\theta})$. If $N$ is large enough, then $C$ follows a $\chi_{\operatorname{dim}(\boldsymbol{\theta})}^{2}$ distribution. So if $C$ takes a value compatible with a $\chi_{\operatorname{dim}(\boldsymbol{\theta})}^{2}$, it is an indication that $N$ has been chosen sufficiently large to do the approximation (7). In our study we found that $N=1000$ was sufficient in all our computations to achieve a $\mathrm{p}$ value of $10 \%$.

It can be noted that the expression (6) could be easily extended to include variable designs between patients as well as explanatory variables.

\subsection{The FIM in the patient-by-patient approach}

Another paradigm for analyzing data is to consider each patient $i$, and thus each vector of individual parameter $\tilde{\boldsymbol{\xi}}^{(i)}$ as a separate entity: this approach is named in the following the patient-by-patient approach. More formally, the model for the system is defined only by expression (1), there are no random effects $\left(\mathbf{u}_{\mathbf{i}} \equiv 0\right)$, and the statistical model is defined for only one patient. The set of parameters can be reduced to $\boldsymbol{\theta}=\left(\left(\phi_{l}\right)_{l \leq p},\left(\sigma_{l}\right)_{l \leq M}\right)$; since there are no random effects and only one patient, the full log-likelihood 
and the full score are $L_{\mathcal{O}}=L_{\mathcal{F}_{i} \mid} \boldsymbol{u}^{(i)}$ and $\boldsymbol{U}_{\mathcal{O}}=\boldsymbol{U}_{\mathcal{F}_{i} \mid \boldsymbol{u}^{(i)}}$ respectively. Concerning the FIM, we can write $\mathcal{I}_{\mathcal{D}, n}(\boldsymbol{\theta})=\mathcal{I}_{\mathcal{D}, i}(\boldsymbol{\theta})=\mathcal{I}_{\mathcal{D}}(\boldsymbol{\theta})$; moreover, by independence of the measurements errors, we have an analytical expression for the FIM, diagonal per block:

$$
\mathcal{I}_{\mathcal{D}}(\boldsymbol{\theta})=\left(\begin{array}{cc}
\mathcal{I}_{\phi \phi} & 0 \\
0 & \mathcal{I}_{\sigma \sigma}
\end{array}\right)
$$

with

$$
\left\{\begin{array}{l}
\mathcal{I}_{\phi \phi}\left(l, l^{\prime}\right)=\sum_{j, m} \frac{1}{\sigma_{m}^{2}} \frac{\partial g_{m}\left(X\left(t_{j m}, \tilde{\boldsymbol{\xi}}^{(i)}\right)\right)}{\partial \phi_{l}} \frac{\partial g_{m}\left(X\left(t_{j m}, \tilde{\boldsymbol{\xi}}^{(i)}\right)\right)}{\partial \phi_{l^{\prime}}}{ }^{T} \\
\mathcal{I}_{\sigma \sigma}(l, l)=\frac{2 n_{l}}{\sigma_{l}^{2}} \text { and } \mathcal{I}_{\sigma \sigma}\left(l, l^{\prime}\right)=0, l^{\prime} \neq l
\end{array}\right.
$$

where the $\frac{\partial g_{m}\left(X\left(t_{j m}, \tilde{\boldsymbol{\xi}}^{(i)}\right)\right)}{\partial \phi_{l}}$ 's are computed using the systems of sensitivity equations (see Appendix).

In our work, we consider that the patient-by-patient approach is the study of patients, independently one from each other, considering that there is no link from one patient to each other. When data for several patients are available, results are often summarized by providing empirical mean, median, or variance of the $n$ estimations, implying a common framework between the patients. These intuitive empiric estimators "summarize" the individual estimates (Davidian and Giltinian, 1995) and are another way to take the between-subject variability. However the asymptotic properties of these estimators, not issued from a maximum likelihood inference (since the "true" model is the population model as defined in the precedent sections) is not known and would require a simulation study. Moreover, this approach requires a practical identifiability for each patient, which is more difficult to get than that required in the population approach where the sample is considered as a whole. That is why it is current in such analyzes to exclude 
several patients (where estimation was not feasible), leading to potential biases in the parameter estimation. Consequently this approach, which is between the patient-by-patient and the population approach, is not studied here and in the following we consider that the patient-by-patient approach implies no link between the patients: it is the study of only one patient.

\section{Presentation of the HIV dynamic model}

\subsection{A mathematical model for HIV dynamics}

Usual models for HIV dynamics involve uninfected lymphocytes T-CD4 cells (CD4), infected CD4 $\left(T^{*}\right)$ and virions $(\mathrm{V})$. This last compartment can be split in two parts: infectious $\left(V_{I}\right)$ and non infectious $\left(V_{N I}\right)$ virus. A proportion $\omega$ of non-infectious virions is generated during the natural history (Chun et al., 1997); the use of a class of antiretroviral, namely the protease inhibitors (Perelson et al., 1996), leads to an increase of $\omega$. One may also include the effect of reverse transcriptase inhibitors which limits cell infection by inhibiting reverse transcription of HIV RNA (through $\eta$ ). Because the activated T-CD4 cells are the main targets of the virus, we distinguish between activated $(\mathrm{T})$ and quiescent cells (Q). Furthermore, there is a strong rationale for incorporating such information in the model as the activation process is probably one of the major cause of CD4 depletion during HIV infection (Grossman et al., 2000). Therefore, we propose the following system 
of non-linear differential equations:

$$
\left\{\begin{array}{l}
\frac{d Q}{d t}=\lambda+\rho T-\alpha Q-\mu_{Q} Q \\
\frac{d T}{d t}=\alpha Q-(1-\eta) \gamma T V_{I}-\rho T-\mu_{T} T \\
\frac{d T^{*}}{d t}=(1-\eta) \gamma T V_{I}-\mu_{T^{*}} T^{*} \\
\frac{d V_{I}}{d t}=\omega \mu_{T^{*}} \pi T^{*}-\mu_{V} V_{I} \\
\frac{d V_{N I}}{d t}=(1-\omega) \mu_{T^{*}} \pi T^{*}-\mu_{V} V_{N I}
\end{array}\right.
$$

where parameters are detailed in Table 1 . We make the assumption that before initiation of antiretroviral treatment the values of the state variables are that of steady state of the system of ODE with $\eta=0$. This assumption implies that the treatment is initiated far from primo-infection. The steady state assumption leads to the following initial conditions (where $t=0$ refers to treatment initiation):

$$
\left\{\begin{array}{l}
Q(0)=\frac{1}{\alpha+\mu_{Q}}\left(\lambda+\frac{\rho \mu_{v}}{\omega \gamma \pi}\right) \\
T(0)=\frac{\mu_{v}}{\omega \gamma \pi} \\
T^{*}(0)=\frac{1}{\mu_{T^{*}}}\left(\frac{\alpha}{\alpha+\mu_{Q}}\left(\lambda+\frac{\rho \mu_{v}}{\omega \gamma \pi}\right)-\frac{\left(\rho+\mu_{T}\right) \mu_{v}}{\omega \gamma \pi}\right) \\
V_{I}(0)=\frac{\alpha \omega \pi}{\mu_{v}\left(\alpha+\mu_{Q}\right)}\left(\lambda+\frac{\rho \mu_{v}}{\omega \gamma \pi}\right)-\frac{\rho+\mu_{T}}{\gamma} \\
V_{N I}(0)=\frac{(1-\omega) \pi}{\mu_{v}}\left(\frac{\alpha}{\alpha+\mu_{Q}}\left(\lambda+\frac{\rho \mu_{v}}{\omega \gamma \pi}\right)-\frac{\mu_{v}\left(\rho+\mu_{T}\right)}{\omega \gamma \pi}\right)
\end{array}\right.
$$

This model belongs to the typical class of non-linear systems of ODE introduced in the literature (Wei et al., 1995; Ribeiro et al., 2002).

\subsection{Values for the parameters}

Parameters were estimated in a previous work (Guedj et al., 2007) using data from an HIV clinical trial (ALBI ANRS 070) comparing different regimen of nucleosides reverse transcriptase inhibitors. Three random effects were retained; the design of this study, not adapted to dynamics models, did not make possible to estimate the $p$ biological parameters and some of them were fixed according to values found in the literature. The value $\boldsymbol{\theta}_{0}$ for the parameters are summarized in Table 1. 
[Table 1 about here.]

The resulting trajectory for the observed components with no random effects and no measurements errors is presented on Figure 1. Figure 2 displays a simulation of a whole sample with $n=100$, taking into account variability due to the random effects and to the measurements errors. Moreover, data under 50 copies. $m l^{-1}$ were censored and represented as equal to 50 in the figures. This illustrates the main advantage of the population approach which enables to consider at the same time a sample of patients sharing common but variables features.

[Figure 1 about here.]

[Figure 2 about here.]

\section{Study of practical identifiability according to observational de- signs and parameters of interest}

The practical identifiability of different set of parameters is studied according to different observational designs. First we study it for the given set of parameters estimated elsewhere. Then we discuss the minimum information required to add the other parameters in the previous set. The results are presented with constant values for the error measurements given in Table 2 . Notice that $\mathcal{I}_{\mathcal{D}, n}(\boldsymbol{\theta})$ is directly linked to the value for the vector $\boldsymbol{\sigma}$ : if only one component was observed $(M=1), \mathcal{I}_{\mathcal{D}, n}(\boldsymbol{\theta})$ would be directly proportional to $\sigma_{1}^{2}$

To compare the practical identifiability between the patient-by-patient and the population approach (illustrated in the following for $n=100$ patients) regarding the practical identifiability of the biological parameters $\phi_{l}$, 
we compute for the population model the restricted efficiency $\phi_{s}(\mathcal{D})$ on the restricted set $\boldsymbol{\theta}_{s}=\left\{\boldsymbol{\theta}-\left\{a_{l l}\right\}_{l=1, \ldots, q}\right\}$, since there are no random effects in the patient-by-patient approach.

Last it can be noted that all the results depend on the value $\boldsymbol{\theta}=\boldsymbol{\theta}_{0}$ chosen for the parameters. We explore to what extent the presented results are robust in a plausible neighborhood of $\boldsymbol{\theta}_{0}$.

\subsection{Potential designs}

Observed components: As explained in section 2.2, we ensure a Gaussian framework of the error measurements by working on transformed observations, namely a log-transformation for HIV-RNA concentration, and the fourth root for the related-CD4 compartments (Thiébaut et al., 2003). We analyze successively five combinations of observable components corresponding to an increase of the available information (see Table 2). The values for the related standard deviations (STD) are either estimation from a previous work or fixed according to biological considerations: we consider that $\sigma_{C D 4 a c t}$ (error measurement made on $T^{0.25}$ or $\left(T+T^{*}\right)^{0.25}$ ) has the same value as for the total CD4 count (i.e. $\left.\sigma_{C D 4 a c t}=0.18\right)$. For infected CD4 $\left(T^{* 0.25}\right)$, we take into account their low number comparing to other CD4. We consider that the relative error measurements for a $T^{*}$-cells concentration of $1 \mathrm{~mm}^{-3}$ should be equivalent to that for a total CD4 count at a median value of $400 \mathrm{~mm}^{-3}$. This leads to $\sigma_{C D \text { inf }}=0.04$. When distinguishing between infectious Virus $V_{I}$ and non-infectious Virus $V_{N I}$, we take the same error measurements than when total virus load $V$ is observed.

Schedules: We compare two different schedules: the schedule S0 is similar to what was used for parameter estimation: measurements are done every 
4 weeks until 24 weeks. The second schedule $\mathbf{S} \mathbf{1}$ is particularly adapted to HIV dynamics analysis. As noted in Perelson (2002), those studies require more intensive measures at the initiation of the therapy, when there is a maximum amount of information on the involved dynamics, particularly the virus load: for $\mathbf{S 1}$, measurements are done every 2 hours until the sixth hour, every 6 hours until day 2 and every day until day 7 like studies focusing on life-span of virions and-or infected cells (Wei et al., 1995; Ho et al., 1995, Perelson et al., 1996; Perelson, 2002). After the first week, the schedule of measurements is the same as in S0.

[Table 2 about here.]

4.2 Identifiability of $\boldsymbol{\theta}=\left(\tilde{\alpha}, \tilde{\lambda}, \tilde{\mu}_{T *}, \tilde{\pi}, \tilde{\mu}_{T}, \tilde{\eta}, a_{\tilde{\alpha}}, a_{\tilde{\lambda}}, a_{\tilde{\mu}_{T *}}\right)$

For $M=1$ (only total virus load is observed), the lowest eigenvalue was less than $10^{-10}$ in the two approaches, leading to very large expected standard deviations, in particular for $\tilde{\pi}$ and $\tilde{\lambda}$. This case is a limit case of theoretical identifiability and the analysis of the corresponding eigenvector suggests to introduce the macroparameter $\tilde{\pi}+\tilde{\lambda}$ (or $\pi \lambda$ equivalently).

For $M>1$, the results are displayed on Table 3. Using the patientby-patient approach with $\mathcal{D}_{G, S}=\left(\mathbf{S 0},{ }^{\prime} V|Q| T+T^{*}\right)$ and more intensive schedule $\mathcal{D}_{G, S}^{\prime}=\left(\mathbf{S} 1,{ }^{\prime} V|Q| T+T^{* \prime}\right)$, the global accuracy on the 8 parameters is improved as expected $\left(\phi\left(\mathcal{D}^{\prime}\right)=36.6\right.$ vs $\left.\phi(\mathcal{D})=27.5\right)$ and the gain of precision is $\mathcal{E}_{\mathcal{D}, \mathcal{D}^{\prime}}=77 \%$ (see expression (4)).

Whatever the chosen approach (patient-by-patient or population), the number of observed components strongly increases the accuracy of the estimation. The improvement is less important when increasing the number of measurements, that is using the intensive schedule S1 rather than S0. For 
instance let us focus on the population model with $\mathcal{D}_{G, S}=\left(\mathbf{S} 0,{ }^{\prime} V|Q| T+T^{* \prime}\right)$. If the schedule of measurements were more intensive (S1), the global accuracy is increased $\left(\phi\left(\mathcal{D}^{\prime}\right)=110.0\right.$ vs $\left.\phi(\mathcal{D})=99.7\right)$. If we keep schedule S0 but if we add the observation of infected cells (S0,' $\left.V|Q| T \mid T^{* \prime}\right)$ the global accuracy is still better $\left(\phi\left(\mathcal{D}^{\prime \prime}\right)=125.7\right)$. With a standard deviation $\sigma_{C D 4 i n f}$ of 0.0014 , the analysis of the correlation matrix shows that the estimator of $\sigma_{C D \text { inf }}$ has a very low correlation with other parameters estimators. Consequently, if we note $\theta_{s}=\left\{\theta-\left\{\sigma_{C D 4 i n f}\right\}\right\}$, then $\phi_{s}\left(\mathcal{D}^{\prime \prime}\right) \simeq \phi\left(\mathcal{D}^{\prime \prime}\right)-\ln \left(0.0014^{-2}\right)=112.5$. The global increase of precision on the 12 remaining parameters is consequently $\mathcal{E}_{\mathcal{D}^{\prime}, \mathcal{D}^{\prime \prime}}=\exp [(112.5-110.0) / 2 * 12]=1.11$, i.e. an accuracy improvement of about $11 \%$.

The comparison between the patient-by-patient and the population approach leads to very clear results. For the baseline model and $\mathcal{D}_{G, S}=\left(\mathbf{S} 0,{ }^{\prime} V|Q| T+\right.$ $\left.T^{* \prime}\right)$ and when focusing on the fixed effect, the global standard errors are reduced by approximately 7.3 fold $(\exp [(59.3-27.5) / 2 * 8]=7.3)$. This explains why we only focus in the following on the population approach.

[Table 3 about here.]

\subsection{Practical identifiability of all parameters}

A further step is to look at the designs that allow the estimation of all biological parameters included in the model (8). We add progressively parameters to the initial model according to the increase of the information. Results are presented in Table 4, omitting the expected standard deviations of the random effects and of the measurement errors.

The focus on $\tilde{\xi}=\left(\tilde{\alpha}, \tilde{\lambda}, \tilde{\mu}_{T *}, \tilde{\pi}, \tilde{\eta}, \tilde{\mu}_{T}, \tilde{r}, \tilde{\gamma}\right)$ with $M=2$ illustrates the difference between practical and theoretical identifiability. Expected standard 
deviation for $\tilde{\gamma}$ and $\tilde{\eta}$ are very close to one for $\mathbf{S}=\mathbf{S} \mathbf{0}$, meaning that even if there is theoretical identifiability (due to the steady state assumption at $t=0$ ), these two parameters cannot be in practice simultaneously estimated. An analysis of the eigenvalues confirms that only the product $\tilde{\gamma}(1-\tilde{\eta})$ could be identifiable. To make these two parameters simultaneously identifiable, one has to use $\mathbf{S 1}$ instead of S0. It can be noted that the steady state condition before initiation of therapy is necessary to have a theoretical identifiability between the two parameters. Moreover, the dramatic drop of the virus load in the first days is highly correlated with $\eta$ and that is why only intensive measurements can measure the specific effect of treatment. However, it can be noted that a classic schedule S0 becomes sufficient if $M=3$. No other parameter can be added to the estimation if $M=2$.

For making other parameters practically identifiable, other components should be observed. To simultaneously estimate $\pi$ and $\mu_{V}$, four or more $(M \geq 4)$ components should be observed through an intensive schedule S1.

With an adequate design, all the parameters are identifiable except $\tilde{\mu_{Q}}$, the transformed death rate of the CD4 quiescent cells $\left(\sigma\left(\tilde{\mu}_{Q}\right)>3\right.$ for all tested designs). This dynamic is low, compared to other involved mechanisms, and a follow-up longer than 24 weeks would be necessary to estimate this parameter.

[Table 4 about here.]

\subsection{Practical identifiability around values chosen for the parameters}

The computation of $\mathcal{I}_{\mathcal{D}}(\boldsymbol{\theta})$ for $\boldsymbol{\theta}=\boldsymbol{\theta}_{0}$ implies that $\boldsymbol{\theta}_{0}$ is known, whereas the study aims precisely at determining if the estimation of $\boldsymbol{\theta}_{0}$ is feasible. This paradox can be overcome in part when studying the variation of $\mathcal{I}_{\mathcal{D}, n}(\boldsymbol{\theta})$ 
in a plausible region around $\boldsymbol{\theta}_{0}$. A natural way is to study the distribution of $\phi(\mathcal{D})$ for $\boldsymbol{\theta} \sim \mathcal{N}\left(\boldsymbol{\theta}_{0} ; \mathcal{I}_{\mathcal{D}}^{-1}\left(\boldsymbol{\theta}_{0}\right)\right)$. A similar approach is used when looking for optimal sequential designs as an alternative to the local planification (Walter and Pronzato, 1997): one can consider an a priori distribution in a Bayesian standing for $\theta$ and looking for $\mathcal{D} *$ maximizing $\mathbb{E}(\phi(\mathcal{D}))$.

Instead of $\phi(\mathcal{D})$, Figure 3 displays the empirical distribution of $\mathcal{E}_{\mathcal{D}}$ obtained with 500 simulations, giving a better idea in term of marginal expected standard deviations. The set of parameters of interest is $\boldsymbol{\theta}=\left(\tilde{\alpha}, \tilde{\lambda}, \tilde{\mu}_{T *}, \tilde{\pi}, \tilde{\mu}_{T}, \tilde{\eta}, a_{\tilde{\alpha}}, a_{\tilde{\lambda}}, a_{\tilde{\mu}_{T *}}\right)$ and $\mathcal{D}=\left(\mathbf{S} 0,{ }^{\prime} V \mid Q+T+T^{*}\right)$ (see Table 3 for results with central values $\left.\boldsymbol{\theta}_{0}\right)$. As it could be hoped, the distribution of $\mathcal{E}_{\mathcal{D}}$ is centered around the value found in $\boldsymbol{\theta}_{0}\left(\mathcal{E}_{\mathcal{D}}\left(\theta_{0}\right)=0.0233\right)$. Moreover, the standard deviation is not large and the values remain of the same order of magnitude than in $\boldsymbol{\theta}_{0}$, meaning that the results given in Table 3 and Table 4 are robust in the domain of the plausible values for $\boldsymbol{\theta}$. Moreover, this could constitute asymptotically a way for assessing the local identifiability as defined in section 2.4.

[Figure 3 about here.]

\section{Conclusions}

In summary, one the main interest of the proposed methodology lies in its ability to compute the FIM as exactly as wanted, which is critical in complex models such as those defined by non-linear ODE, treated in a population context. We have proposed a method to compute the FIM giving the opportunity to evaluate the practical identifiability and to compare it through different observational designs. Although the conclusion might depend partly on the biological model, the application of the method has provided interesting results, such as outlining the benefit of increasing the number of com- 
ponents measured. Therefore, the availability of assays able to distinguish infected and non infected cells (Patterson et al., 1998) as well as infectious and non infectious viruses (Rusert et al., 2004) may substantially improve the identifiability of model parameters and thus the usefulness of HIV dynamics model to analyze real data. Of note, the impact of increasing the measurements schedule intensity depends also on the parameters of interest. For instance, if we wish to estimate simultaneously the free virion clearance and the number of virus produced by an infected cell, the design should be adapted to the rapid dynamics of these components, i.e. schedules with more frequent measurements should be favored. All the same, an intensive schedule is necessary to distinguish the infectivity of the virus and the in vivo treatment efficacy.

We have quantified the improvement of the population approach compared to the patient-to-patient approach. Although it may seem obvious to some that there is a large improvement in the population approach, the computationally simpler patient-by patient approach is still widely used; however for estimating a reasonable number of parameters, intensive measurements schedules are necessary. Most often, ethics and cost considerations make such intensive measurements schedules difficult to obtain, so the population approach is the method of choice.

As noted by one of the referees, a Bayesian inference, taking advantage of the MCMC techniques, have been widely used in complex models (Auranen et al., 2000; Cauchemez et al., 2006) and can be used in particular for population models based on ODE systems (Putter, 2002). The maximum likelihood method, when feasible, may retain some advantages however in 
term of computation time and reliability of convergence criteria. A comparison of the two approaches is difficult, in particular because of the use of a priori information in the Bayesian approach: it may be considered as an advantage, especially if such information really exists and comes for instance from expert knowledge, but may also be considered a drawback because of its subjective nature and the risk of influencing too much the final result by inappropriate choice of the prior. These differences also have concrete implications: in the Bayesian approach, the study of the practical identifiability requires to evaluate by simulation the expectation of the posterior variance-covariance matrix of the parameters of interest (Han and Chaloner, 2004) and different criteria have been proposed to compare designs (Walter and Pronzato, 1997). Another extension of the present work would be to find optimal designs (Atkinson and Donev, 1992; Mentré et al., 1997) taking into account or not the cost issue due to the increase of the number of measurements or due to the use of new type of quantification.

Our methodology is introduced by defining an i.i.d framework for the patients. For sake of clarity, we did not take into account the possibility to include explanatory variables like treatment group (Guedj et al., 2007) or pharmacokinetics data (Huang and $\mathrm{Wu}, 2006$ ) but the computation of the FIM would remain very close to what was presented here.

In conclusion, our primary interest is to propose a methodology to check the practical identifiability when designing a study aiming at estimating parameters of a dynamical model. In addition, we provide in this work an order of magnitude for the relative errors that can be hoped in typical HIV dynamics models according to usual designs. 


\section{REFERENCES}

Atkinson, A. and Donev, A. (1992). Optimum Experimental Designs. Oxford University Press.

Audoly, S., Bellu, G., D’Angio, L., Saccomani, M. and Cobelli, C. (2001). Global Identifiability of Nonlinear Models of Biological Systems. IEEE Transactions on biomedical engineering 48, 55-65.

Auranen, K., Arjas, E., Leino, T. and Takala, A. (2000). Transmission of Pneumococcal Carriage in Families: A Latent Markov Process Model for Binary Longitudinal Data. Journal of the American Statistical Association 95.

Banks, H., Grove, S., Hu, S. and Ma, Y. (2005). A hierarchical Bayesian approach for parameter estimation in HIV models. Inverse Problems 21, $1803-1822$.

Bortz, D. and Nelson, P. (2006). Model Selection and Mixed-Effects Modeling of HIV Infection Dynamics. Bulletin of Mathematical Biology 68, 20052025 .

Box, G. E. P. and Draper, N. R. (1959). A basis for the selection of a response surface design. Journal of the American Statistical Association $\mathbf{5 4}, 622-654$.

Cauchemez, S., Temime, L., Guillemot, D., Varon, E., Valleron, A., Thomas, G. and Boëlle, P. (2006). Investigating Heterogeneity in Pneumococcal Transmission: A Bayesian MCMC Approach Applied to a Follow-up of Schools. Journal of the American Statistical Association 101, 946-958.

Chun, T. W., Carruth, L., Finzi, D., Shen, X., DiGiuseppe, J. A., Taylor, H., 
Hermankova, M., Chadwick, K., Margolick, J., Quinn, T. C., Kuo, Y. H., Brookmeyer, R., Zeiger, M. A., Barditch-Crovo, P. and Siliciano, R. F. (1997). Quantification of latent tissue reservoirs and total body viral load in HIV-1 infection. Nature 387, 183-188.

Commenges, D., Jacqmin-Gadda, H., Proust, C. and Guedj, J. (2006). A Newton-like algorithm for likelihood maximization: the robust variance scoring algorithm. arXiv:math.ST/0610402 .

Davidian, M. and Giltinian, D. M. (1995). Nonlinear Models for Repeated Measurements Data. Chapman and Hall, London.

Fedorov, V. V. (1972). Theory of Optimal Experiments. Academic Press.

Genz, A. and Keister, B. D. (1996). Fully symmetric Interpolatory Rules for Multiple Integrals over Infinite Regions with Gaussian Weight. Journal of Computational and Applied Mathematics 71, 299-311.

Grossman, Z., Meier-Schellersheim, M., Sousa, A. E., Victorino, R. M. M. and Paul, W. E. (2000). CD4+ T-cell depletion in HIV infection: Are we closer to understanding the cause? Nature Medicine 8, 319-323.

Guedj, J., Thibaut, R. and Commenges, D. (2007). Maximum Likelihood Estimation in dynamical models of HIV. Biometrics (in press).

Han, C. and Chaloner, K. (2004). Bayesian experimental design for nonlinear mixed-effects models with application to HIV dynamic. Biometrics 60, $25-33$.

Ho, D. D., Neumann, A. U., Perelson, A. S., Chen, W., Leonard, J. M. and Markowitz, M. (1995). Rapid turnover of plasma virions and CD4 lymphocytes in HIV-1 infection. Nature 373, 123-126.

Holmberg, A. (1982). On the practical identifiability of microbial growth 
models incorporating Michaelis-Menten type nonlinearities. Mathematical Biosciences 62, 23-43.

Huang, Y., Liu, D. and Wu, H. (2006). Hierarchical Bayesian methods for estimation of parameters in a longitudinal HIV dynamic system. Biometrics 63, 413-423.

Huang, Y. and Wu, H. (2006). A bayesian approach for estimating antiviral efficacy in HIV dynamic models. Journal of Applied Statistics 33, 155174.

Julien, S., Barbary, J. and Lessard, P. (1997). Theoretical and practical identifiability of a reduced order model in an activated sludge process doing nitrification and denitrification. Water Science 8 Technology 37, 309-316.

Kiefer, J. (1974). General equivalence theory for optimum designs (approximate theory). Annals of Statistics 2, 849-879.

Knight, K. (2001). Mathematical Statistics. Chapman \& Hall/CRC.

Louis, T. (1982). Finding the observed Information matrix when using the EM algorithm. Journal of the Royal Statistical Society. Series B 44, $226-233$.

Mclean, A. R. and Michie, C. A. (1995). in vivo estimates of division and death rates of human t lymphocytes. Proceedings of the National Academy of Sciences of the United States of America 92, 3707-3711.

Mentré, F., Mallet, A. and Baccar, D. (1997). Optimal design in randomeffects regression models. Biometrika 84, 429-442.

Patterson, B. K., Mosiman, V. L., Cantarero, L., Furtado, M., Bhattacharya, M. and Goolsby, C. (1998). Detection of HIV-RNA-positive monocytes 
in peripheral blood of HIV-positive patients by simultaneous flow cytometric analysis of intracellular HIV RNA and cellular immunophenotype. Cytometry 31, 265-274.

Perelson, A. S. (2002). Modelling viral and immune system dynamics. Nature Reviews Immunology 2, 28-36.

Perelson, A. S., Neumann, A. U., Markowitz, M., Leonard, J. M. and Ho, D. (1996). Viral dynamics in human immunodeficiency virus type 1 infection. Science 271, 1582-1586.

Petersen, B., Gernaey, K. and Vanrolleghem, P. A. (2001). Practical identifiability of model parameters by combined respirometric-titrimetric measurements. Water Science and Technology 43, 347-355.

Piatak, M., Saag, M., Yang, L. C., Clark, S. J., Kappes, J. C., Luk, K. C., Hahn, B. H., Shaw, G. M. and Lifson, J. D. (1993). High levels of HIV-1 in plasma during all stages of infection determined by competitive PCR. Science 259, 1749-1754.

Pinheiro, J. C. and Bates, D. M. (2000). Mixed-Effects Models in S and $S$-PLUS. Springer, London.

Pohjanpalo, H. (1978). System identifiability based on the power series expansion of the solution. Mathematical Biosciences 41.

Putter, H., Heisterkamp, S. H., Lange, J. M. A. and deWolf, F. (2002). A Bayesian approach to parameter estimation in HIV dynamic models. Statistics in Medicine 21, 2199-2214.

Radhakrishnan, K. and Hindmarsh, A. C. (1993). Description and use of lsode, the livermore solver for ordinary differential equations. LLNL Report UCR-ID-113855 Livermore, CA 4. 
Ramratnam, B., Bonhoeffer, S., Binley, J., Hurley, A., Zhang, L., Mittler, J. E., Markowitz, M., Moore, J. P., Perelson, A. S. and Ho, D. D. (1999). Rapid production and clearance of HIV-1 and hepatitis $\mathrm{C}$ virus assessed by large volume plasma apheresis. The Lancet 354, 1782-1786.

Retout, S., Dufull, S. and Mentr, F. (2001). Development and implementation of the population Fisher information matrix for the evaluation of population pharmacokinetic designs. Computer Methods and Programs in Biomedicine 65, 141-151.

Ribeiro, R. M., Mohri, H., Ho, D. D. and Perelson, A. S. (2002). In vivo dynamics of T cell activation, proliferation, and death in HIV-1 infection: Why are CD4 but not CD8 T cells depleted? Proceedings of the National Academy of Sciences 24, 15572-15577.

Rothenberg, T. J. (1971). Identification in parametric models. Econometrica 39, $577-591$.

Rusert, P., Fischer, M., Joos, B., Leemann, C., Kuster, H., Flepp, M., Bonhoeffer, S., Gunthard, H. F. and Trkola, A. (2004). Quantification of infectious HIV-1 plasma viral load using a boosted in vitro infection protocol. Virology 326, 113-129.

Stafford, M. A., Corey, L., Cao, Y., Daar, E. S., Ho, D. D. and Perelson, A. S. (2000). Modeling plasma virus concentration during primary HIV infection. Journal of Theoretical Biology 203, 285-301.

Thiébaut, R., Guedj, J., Jacqmin-Gadda, H., Chêne, G., Trimoulet, P., Neau, D. and Commenges, D. (2006). Estimation of dynamical model parameters taking into account undetectable marker values. BMC Medical Research Methodology 6, 1-10. 
Thiébaut, R., Jacqmin-Gadda, H., Leport, C., Katlama, C., D., C., Le Moing, V., Morlat, P., Chene, G. and the APROCO Study Group (2003). Bivariate longitudinal model for the analysis of the evolution of HIV RNA and CD4 cell count in HIV infection taking into account left censoring of HIV RNA measures. Journal of Biopharmaceutical Statistics 13, 271-282.

Vajda, S., Rabitz, H., Walter, E. and Lecourtier, Y. (1989). Qualitative and quantitative identifiability analysis of nonlinear chemical kinetic models. Chemical Engineering Communication 83, 191-219.

Walter, E. and Pronzato, L. (1997). Identification of Parametric Models from Experimental Data. Springer-Verlag.

Wei, X., Ghosh, S. K., E., T. M., Johnson, V. A., Emini, E. A., Deutsch, P., Lifson, J. D., Bonhoeffer, S., Nowak, N. A., Hahn, B. H., Saag, M. S. and Shaw, G. M. (1995). Viral dynamics in human immunodeficiency virus type 1 infection. Nature $373,117-122$.

Wu, H. (2005). Statistical methods for HIV dynamic studies in AIDS clinical trials. Statistical Methods in Medical Research 14, 1-22.

Xia, X. and Moog, C. H. (2003). Identifiability of nonlinear systems with applications to HIV/AIDS models. IEEE transactions on automatic control 48, 330-336.

\section{Appendix A}

Computation of the individual score in a system of $O D E$

For simplicity, we assume in this section that there is no censored data.

Three types of parameters can be distinguished: biological parameters $\tilde{\xi}_{l}$, terms $a_{l l^{\prime}}$ (see equation (2)) and standard deviation of the measurement 
errors $\sigma_{l}$ as defined in equation (3). Consequently, one can get three different expressions for the full score itself. For subject $i$ at the current point $\boldsymbol{\theta}=$ $\left(\left(\tilde{\xi}_{l}\right)_{l \leq p},\left(a_{l l}\right)_{l \leq q},\left(\sigma_{l}\right)_{l \leq M}\right)$ the components of the full score can be written as follows:

$$
\begin{aligned}
& U_{\mathcal{F}_{i} \mid \boldsymbol{u}^{(i)}}^{\left(\phi_{l}\right)}(\boldsymbol{\theta})=\frac{\partial L_{\mathcal{F}_{i} \mid \boldsymbol{u}^{(i)}}}{\partial \tilde{\xi}_{l}^{(i)}}=\sum_{m \leq M, j \leq n_{i m}} \frac{1}{\sigma_{m}^{2}} \frac{\partial g_{m}\left(X\left(t_{i j m}, \tilde{\boldsymbol{\xi}}^{(i)}\right)\right)}{\partial \tilde{\boldsymbol{\xi}}^{(i)}}\left[Y_{i j m}-g_{m}\left(X\left(t_{i j m}, \tilde{\boldsymbol{\xi}}^{(i)}\right)\right)\right] \\
& U_{\mathcal{F}_{i} \mid \boldsymbol{u}^{(i)}}^{\left(a_{l l}\right)}(\boldsymbol{\theta})=\frac{\partial L_{\mathcal{F}_{i} \mid \boldsymbol{u}^{(i)}}}{\partial a_{l l}}=\sum_{m \leq M, j \leq n_{i m}} \frac{1}{\sigma_{m}^{2}}\left(Y_{i j m}-g_{m}\left(\boldsymbol{X}\left(t_{i j m}, \tilde{\boldsymbol{\xi}}^{(i)}\right)\right)\right)\left(u_{l}^{(i)} \frac{\partial g_{m}\left(\boldsymbol{X}\left(t_{i j m}, \tilde{\boldsymbol{\xi}}^{(i)}\right)\right)}{\partial \tilde{\xi}_{l}^{(i)}}\right) \\
& U_{\mathcal{F}_{i} \mid \boldsymbol{u}^{(i)}}^{\left(\sigma_{l}\right)}(\boldsymbol{\theta})=\frac{\partial L_{\mathcal{F}_{i} \mid \boldsymbol{u}^{(i)}}}{\partial \sigma_{l}}=\sum_{j \leq n_{i l}} \frac{\left(Y_{i j l}-g_{l}\left(\boldsymbol{X}\left(t_{i j l}, \tilde{\boldsymbol{\xi}}^{(i)}\right)\right)\right)^{2}}{\sigma_{l}^{3}}-\frac{n_{i l}}{\sigma_{l}}
\end{aligned}
$$

For computing the scores we thus have to compute:

$$
\frac{\partial g_{m}\left(\boldsymbol{X}\left(t, \tilde{\boldsymbol{\xi}}^{(i)}\right)\right)}{\partial \tilde{\xi}_{l}^{(i)}}=\sum_{k \leq K} \frac{\partial g_{m}\left(\boldsymbol{X}\left(t, \tilde{\boldsymbol{\xi}}^{(i)}\right)\right)}{\partial X^{(k)}} \frac{\partial X^{(k)}\left(t, \tilde{\boldsymbol{\xi}}^{(i)}\right)}{\partial \tilde{\boldsymbol{\xi}}^{(i)}}
$$

the computation of the full score requires to solve numerically the $p$ systems of sensitivity equations $\frac{\partial X^{(k)}\left(t, \tilde{\boldsymbol{\xi}}^{(i)}\right)}{\partial \tilde{\xi}_{l}^{(i)}}$. The systems of sensitivity equations are solved with the same routine (Radhakrishnan and Hindmarsh, 1993) than what was used for the original system (see section 2.3). 

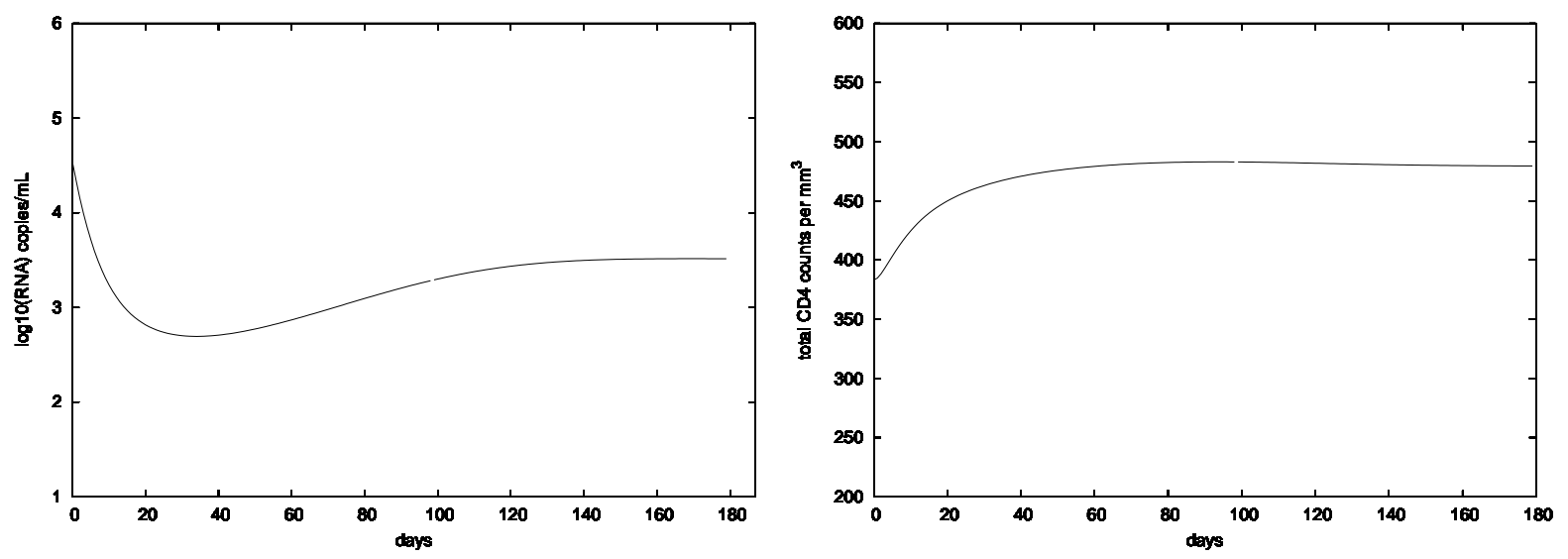

Figure 1. Simulated trajectories for the virus load (left) and the total CD4 counts (right) after antiretroviral treatment initiation when the random effects and the error measurements are set to be null. Values for the parameters are those given in Table 1. 

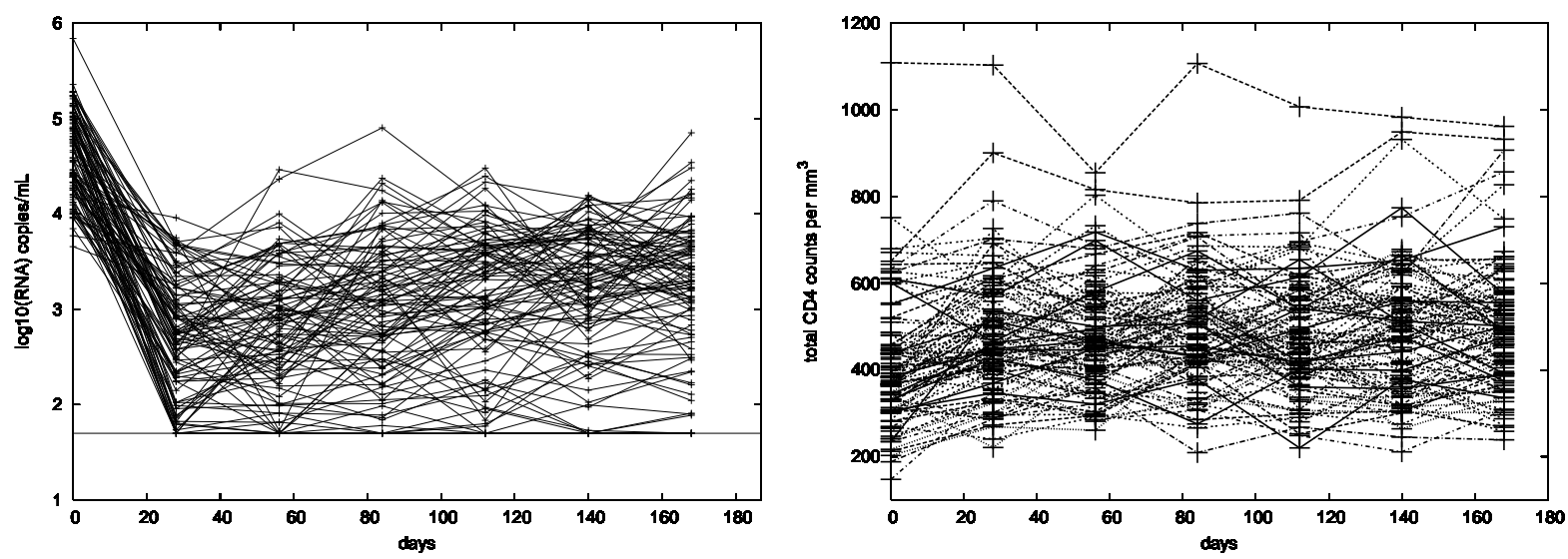

Figure 2. 100 simulated trajectories for the virus load (left) and the total CD4 counts (right) The trajectories for the biomarkers share common features but may vary between the (simulated) patients since each individual value of $\alpha, \lambda$ and $\mu_{T *}$ are the realization of random variables (with variances $\left.a_{\tilde{\alpha}}, a_{\tilde{\lambda}}, a_{\tilde{\mu}_{T *}}\right)$. Moreover we add to the simulated trajectories fluctuations due to the error measurements (with variances $\sigma_{C V}$ and $\sigma_{C D 4}$ ) and we censure data of virus load lower than the level of detection $\left(1.7 \log _{10} / \mathrm{ml}\right)$; the design of the observation is $\mathbf{S 0}$. 


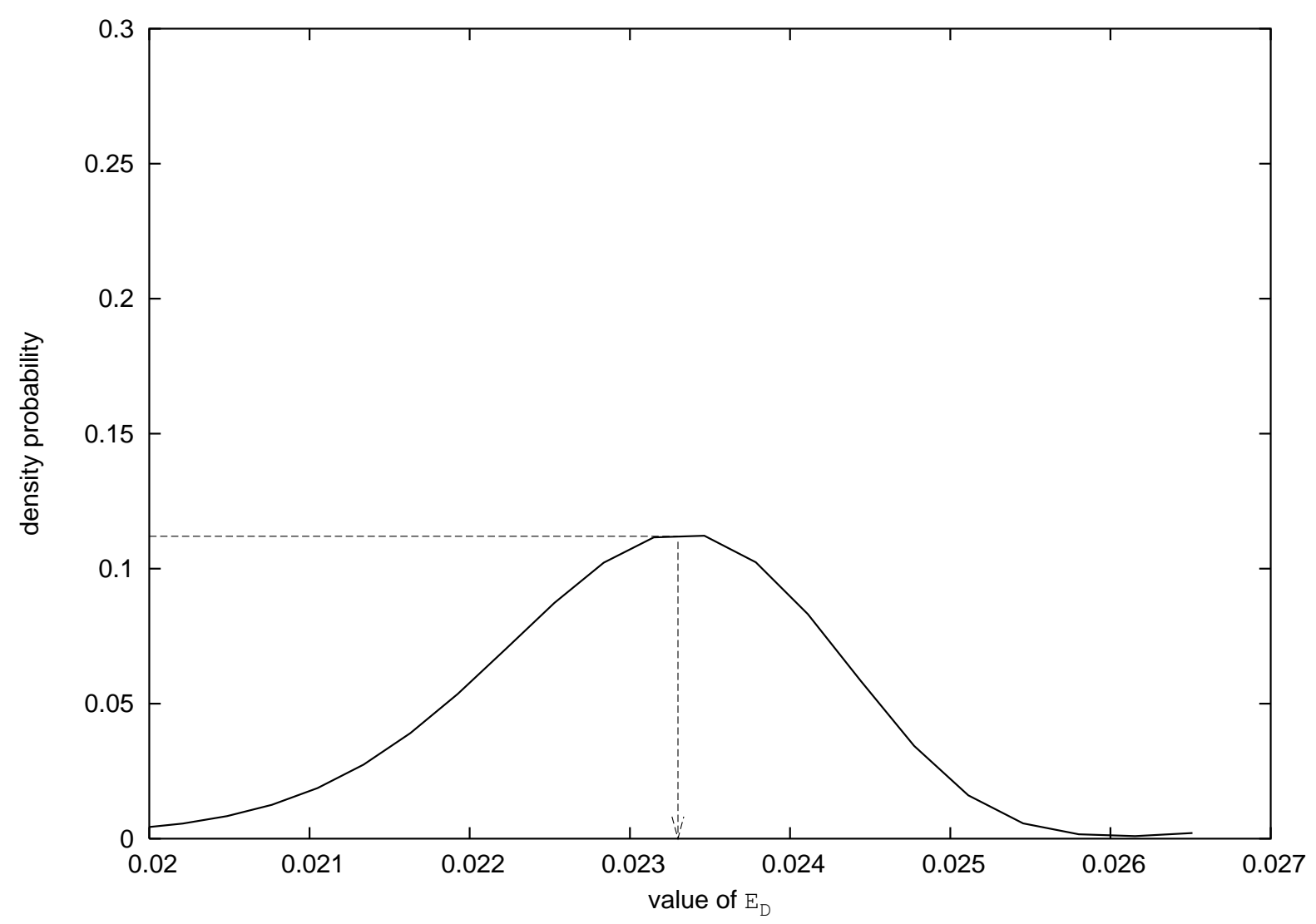

Figure 3. Empirical distribution of $\mathcal{E}_{\mathcal{D}}(\theta)$. The dot line corresponds to the result obtained for the central parameter value $\theta_{0}$ given in Table 3 . The smoothness of the empirical distribution is a good indicator that the results provided for a particular value $\boldsymbol{\theta}=\boldsymbol{\theta}_{0}$ would remain stable in a reasonable neighborhood around $\boldsymbol{\theta}_{0}$. 


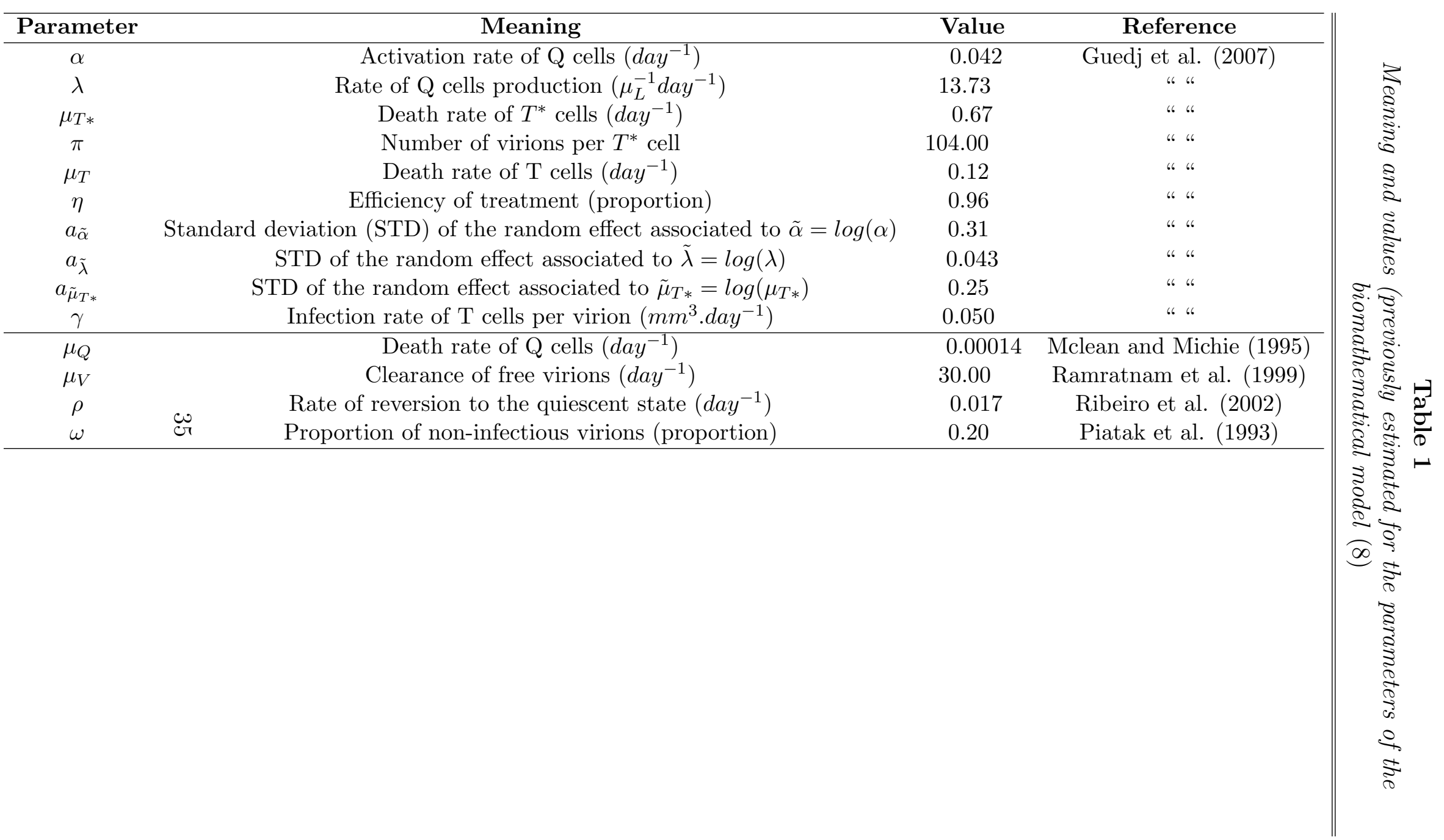


Table 2: Observed components considered regarding the number of available markers $M$

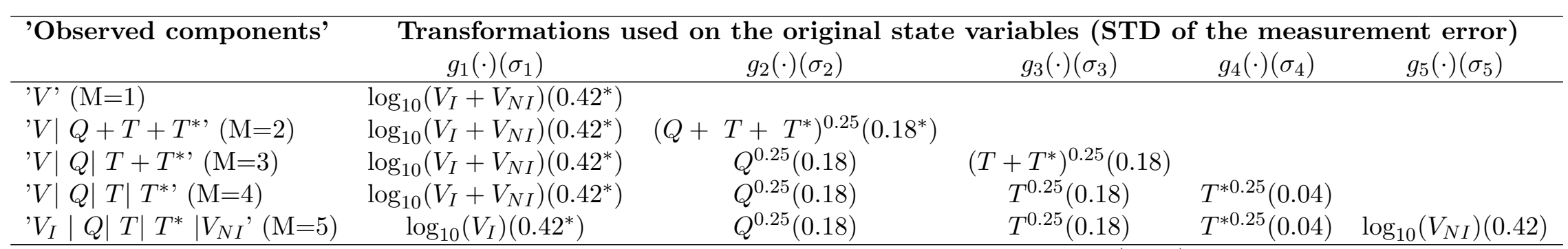

* these values come from estimation performed in Guedj et al. (2007) 


\begin{tabular}{|c|c|c|c|c|c|c|c|c|c|c|c|c|c|}
\hline \multirow[t]{2}{*}{ Design $\mathcal{D}_{S, G}$} & \multirow[b]{2}{*}{$\tilde{\alpha}$} & \multicolumn{7}{|c|}{ Expected standard deviation } & & \multirow{3}{*}{\multicolumn{2}{|c|}{$\phi_{s}(\mathcal{D}) \quad \phi(\mathcal{D})$}} & \multirow[t]{2}{*}{$\mathcal{E}_{\mathcal{D}}$} & \multirow{3}{*}{ 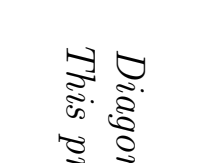 } \\
\hline & & $\tilde{\lambda}$ & $\mu_{\tilde{T} *}$ & $\tilde{\pi}$ & $\tilde{\mu_{T}}$ & $\tilde{\eta}$ & $a_{\tilde{\alpha}}$ & $a_{\tilde{\lambda}}$ & $a_{\tilde{\mu}_{T *}}$ & & & & \\
\hline \multicolumn{11}{|c|}{ patient-by-patient approach } & & & \\
\hline$\overline{\mathbf{S} \mathbf{S 0},{ }^{\prime} V \mid Q+T+T^{*}}$ & 1.25 & 1.04 & 0.85 & 1.04 & 0.74 & 0.76 & - - & - - & - & - & 27.5 & 0.18 & \\
\hline $\mathbf{S} 1,{ }^{\prime} V \mid Q+T+T^{*}$, & 0.76 & 0.67 & 0.35 & 0.62 & 0.38 & 0.33 & - & - & - & - & 36.6 & 0.10 & \\
\hline S0, ${ }^{\prime} V|Q| T+T^{*}$, & 0.58 & 0.63 & 0.79 & 0.41 & 0.69 & 0.59 & - & - & - & - & 39.4 & 0.11 & \\
\hline $\mathbf{S} 1,{ }^{\prime} V|Q| T+T^{*}$, & 0.20 & 0.21 & 0.27 & 0.11 & 0.27 & 0.18 & - & - & - & - & 50.4 & 0.06 & \\
\hline $\mathbf{S} 1,{ }^{\prime} V|Q| T \mid T^{*}$, & 0.070 & 0.063 & 0.040 & 0.084 & 0.065 & 0.088 & - & - & - & - & 67.0 & 0.035 & \\
\hline \multicolumn{13}{|c|}{ Population approach } & 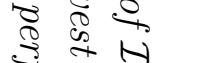 \\
\hline S0, ${ }^{\prime} V \mid Q+T+T^{*}$, & 0.13 & 0.11 & 0.094 & 0.11 & 0.080 & 0.083 & 0.023 & 0.0052 & 0.036 & 59.3 & 82.7 & 0.023 & \\
\hline $\mathbf{S} 1,{ }^{\prime} V \mid Q+T+T^{*}$, & 0.079 & 0.064 & 0.042 & 0.059 & 0.038 & 0.038 & 0.022 & 0.0047 & 0.025 & 66.2 & 92.2 & 0.015 & D \\
\hline So, ${ }^{\prime} V|Q| T+T^{*}$, & 0.068 & 0.067 & 0.085 & 0.042 & 0.059 & 0.073 & 0.024 & 0.0054 & 0.039 & 74.6 & 99.7 & 0.015 & $\tilde{\Xi}$ \\
\hline $\mathbf{S} 1,{ }^{\prime} V|Q| T+T^{*}$, & 0.038 & 0.024 & 0.036 & 0.012 & 0.028 & 0.021 & 0.021 & 0.0043 & 0.024 & 83.7 & 110.0 & 0.010 & క \\
\hline S0, ${ }^{\prime} V \underset{+}{\phi} Q|T| T^{*}$ & 0.024 & 0.014 & 0.027 & 0.017 & 0.012 & 0.019 & 0.014 & 0.0028 & 0.018 & 97.1 & 125.7 & 0.0079 & i \\
\hline & & & & & & & & & & & & & 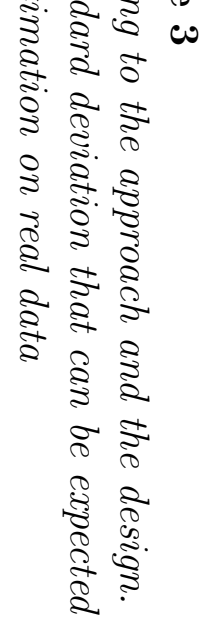 \\
\hline
\end{tabular}




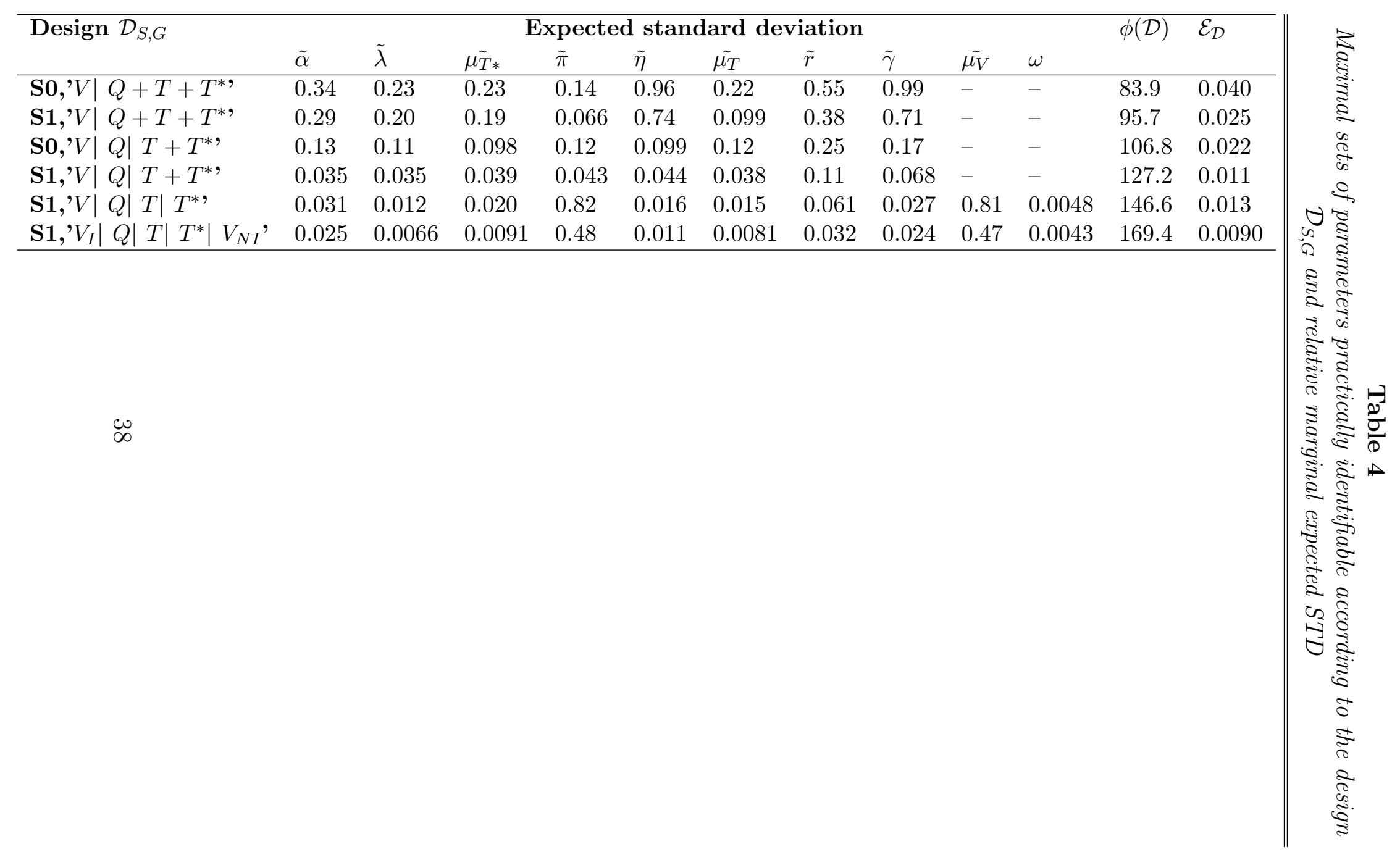

